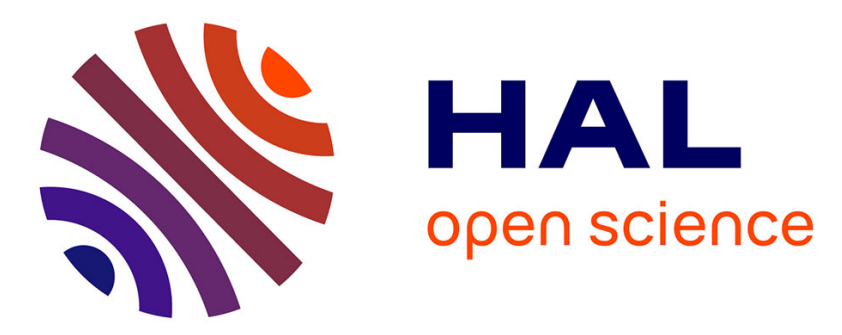

\title{
Channel Estimation for MIMO-OFDM Systems in Fast Time-Varying Environments
}

\author{
Hussein Hijazi, Eric Pierre Simon, M. Lienard, Laurent Ros
}

\section{To cite this version:}

Hussein Hijazi, Eric Pierre Simon, M. Lienard, Laurent Ros. Channel Estimation for MIMO-OFDM Systems in Fast Time-Varying Environments. ISCCSP 2008 - 4th International Symposium on Communications, Control and Signal Processing, Mar 2010, Limassol, Cyprus. 5 p., 10.1109/ISCCSP.2010.5463392 . hal-00539137

\section{HAL Id: hal-00539137 https://hal.science/hal-00539137}

Submitted on 24 Nov 2010

HAL is a multi-disciplinary open access archive for the deposit and dissemination of scientific research documents, whether they are published or not. The documents may come from teaching and research institutions in France or abroad, or from public or private research centers.
L'archive ouverte pluridisciplinaire HAL, est destinée au dépôt et à la diffusion de documents scientifiques de niveau recherche, publiés ou non, émanant des établissements d'enseignement et de recherche français ou étrangers, des laboratoires publics ou privés. 


\title{
Channel Estimation for MIMO-OFDM Systems in Fast Time-Varying Environments
}

\author{
Hussein Hijazi ${ }^{1}$, Eric Pierre Simon ${ }^{1}$, Martine Liénard ${ }^{1}$ and Laurent Ros ${ }^{2}$ \\ ${ }^{1}$ Laboratoire TELICE, U.S.T.L. Lille 1 - IEMN/UMR 8520 Bâtiment P3 59655 Villeneuve d'Ascq - FRANCE \\ ${ }^{2}$ GIPSA-lab, Department Image Signal, BP 46 - 38402 Saint Martin d'Hères - FRANCE \\ hussein.hijazi@hotmail.fr, eric.simon@univ-lille1.fr, martine.lienard@univ-lille1.fr, laurent.ros@gipsa-lab.inpg.fr
}

\begin{abstract}
A channel estimation algorithm for MIMO-OFDM systems in Fast Time-Varying Environments is proposed. The channel estimation function is based on the equivalent discretetime channel taps or on the physical propagation channel parameters. To handle rapid variations of channels within a transmission block, we approximate the channel by a basis expansion model (BEM). Based on the Jakes process, an auto-regressive (AR) model of the BEM coefficients dynamics is built, making it possible to estimate and track the BEM coefficients using Kalman filter. Hence, the channel matrix is easily computed, and the data symbol is detected with free ICI . Our claims are supported by theoretical analysis and simulation results, which are obtained considering Jakes' channels with high Doppler spreads.
\end{abstract}

Index Terms-MIMO systems, OFDM, time-varying Rayleigh channels, channel estimation, Kalman filter.

\section{INTRODUCTION}

Multiple-Input-Multiple-Output (MIMO) antennas with Orthogonal Frequency Division Multiplexing (OFDM) provide high data rates and are robust to multi-path delay in wireless communications. Channel parameters are required for diversity combining, coherent detection and decoding. Therefore, channel estimation is essential in MIMO-OFDM system design. Various OFDM channel estimation schemes have been proposed in the literature, mostly for single antenna systems [8] [12] [14] [9] [10]. For MIMO-OFDM systems, most of the channel estimation schemes have focused on pilot-assisted approaches [1] [3] [4], based on a quasi-static fading model that allows the channel to be invariant within a MIMO-OFDM block. However, in fast fading channels, the time-variation of the channel within a MIMO-OFDM block results in a loss of subcarrrier orthogonality which leads to intercarrier interference (ICI) [8] [2]. To support high speed mobile channels, the channel time-variation within a block must be considered. Channel estimation can be summarized by two approaches. The first one is to estimate the equivalent discretetime channel taps [2] [12] whereas the second approach is to directly estimate the physical propagation channel parameters such as multi-path delays and multi-path complex gains [14] [8]. In Radio-Frequencies transmission, the delays are quasi invariant over several MIMO-OFDM blocks [11] [9] whereas the complex gains may change significantly, even within one MIMO-OFDM block. Exploiting the channel nature and assuming the availability of delay information, a lot of methods estimate the multi-path complex gains [8] [10] [9]. There are more channel parameters in fast fading channels than in quasi- static fading channels. Therefore, in single antenna systems, many existing works resort to the basis expansion model (BEM) in order to estimate the time-variation of the channel taps or the multi-path complex gains within a block [14] [8]. The BEM methods are discrete Karhunen-Love BEM (DKL$\mathrm{BEM}$ ), discrete prolate spheroidal BEM (PS-BEM), complexexponential BEM (CE-BEM), generalized CE-BEM (GCEBEM) and polynomial BEM (P-BEM).

In this paper, we present a new iterative algorithm for joint Rayleigh channel (taps or complex gains) estimation and data recovery in fast time-varying environments. After approximated the time-variation of the channel by a BEM, an autoregressive (AR) model of the BEM coefficients dynamics is built based on the Jakes process. Hence, the BEM coefficients are estimated and tracked by using the Kalman filter. In order to perform BEM coefficients estimation, we use the estimate along with the channel matrix output to recover the transmitted data. One can, in turn, use the detected data along with pilots to enhance the BEM coefficients estimate giving rise to an iterative technique for channel estimation and data recovery. The data detection is performed by using the MMSE-equalizer [13] or the QR-equalizer [8] [7].

This paper is organized as follows: Section II introduces the MIMO-OFDM system and the BEM modeling. Section III describes the AR model for the BEM coefficients and the Kalman filter. Section IV covers the algorithm for joint channel estimation and data recovery. Section $\mathrm{V}$ presents the simulations results which validate our technique. Finally, our conclusions are presented in Section VI.

The notations adopted are as follows: Upper (lower) bold face letters denote matrices (column vectors). $[\mathbf{x}]_{k}$ denotes the $k$ th element of the vector $\mathbf{x}$, and $[\mathbf{X}]_{k, m}$ denotes the $[k, m]$ th element of the matrix $\mathbf{X}$. We will use the matlab notation $\mathbf{X}_{\left[k_{1}: k_{2}, m_{1}: m_{2}\right]}$ to extract a submatrix within $\mathbf{X}$ from row $k_{1}$ to row $k_{2}$ and from column $m_{1}$ to column $m_{2}$. $\mathbf{I}_{N}$ is a $N \times N$ identity matrix and $\mathbf{0}_{N}$ is a $N \times N$ matrix of zeros. $\operatorname{diag}\{\mathbf{x}\}$ is a diagonal matrix with $\mathbf{x}$ on its main diagonal and blkdiag $\{\mathbf{X}, \mathbf{Y}\}$ is a block diagonal matrix with the matrices $\mathbf{X}$ and $\mathbf{Y}$ on its main diagonal. The superscripts $(\cdot)^{T},(\cdot)^{*}$ and $(\cdot)^{H}$ stand respectively for transpose, conjugate and Hermitian operators. $\operatorname{Tr}(\cdot)$ and $\mathrm{E}[\cdot]$ are respectively the determinant and expectation operations. $J_{0}(\cdot)$ is the zeroth-order Bessel function of the first kind. 


\section{MIMO-OFDM SySTEM AND CHANNEL Models}

\section{A. MIMO-OFDM System Model}

Consider a MIMO-OFDM system with $N_{T}$ transmit antennas, $N_{R}$ receive antennas, $N$ sub-carriers, and a cyclic prefix length $N_{g}$. The duration of a MIMO-OFDM block is $T=v T_{s}$, where $T_{s}$ is the sampling time and $v=N+N_{g}$. Let $\mathbf{x}_{(n)}=$ $\left.\left[\mathbf{x}_{(1, n)}^{T}, \mathbf{x}_{(2, n)}^{T}, \ldots, \mathbf{x}_{\left(N_{T}, n\right)}^{T}\right]\right]^{T}$ be the $n$th transmitted MIMOOFDM block, where $\mathbf{x}_{(t, n)}=\left[x_{(t, n)}\left[-\frac{N}{2}\right], x_{(t, n)}\left[-\frac{N}{2}+\right.\right.$ $\left.1], \ldots, x_{(t, n)}\left[\frac{N}{2}-1\right]\right]^{T}$ is the $n$th transmitted OFDM symbol by the $t$ th transmit antenna and $\left\{x_{(t, n)}[b]\right\}$ are normalized symbols (i.e., $\mathrm{E}\left[x_{(t, n)}[b] x_{(t, n)}^{*}[b]\right]=1$ ). After transmission over a multi-path Rayleigh channel, the $n$th received MIMOOFDM block $\left.\mathbf{y}_{(n)}=\left[\mathbf{y}_{(1, n)}^{T}, \mathbf{y}_{(2, n)}^{T}, \ldots, \mathbf{y}_{\left(N_{R}, n\right)}^{T}\right)\right]^{T}$, where $\mathbf{y}_{(r, n)}=\left[y_{(r, n)}\left[-\frac{N}{2}\right], y_{(r, n)}\left[-\frac{N}{2}+1\right], \ldots, y_{(r, n)}\left[\frac{N}{2}-1\right]\right]^{T}$ is the $n$th received OFDM symbol by the $r$ th receive antenna, is given by [9] [12]:

$$
\mathbf{y}_{(n)}=\mathbf{H}_{(n)} \mathbf{x}_{(n)}+\mathbf{w}_{(n)}
$$

where $\left.\mathbf{w}_{(n)}=\left[\mathbf{w}_{(1, n)}^{T}, \mathbf{w}_{(2, n)}^{T}, \ldots, \mathbf{w}_{\left(N_{R}, n\right)}^{T}\right]\right]^{T}$, with $\mathbf{w}_{(r, n)}=$ $\left[w_{(n)}\left[-\frac{N}{2}\right], w_{(n)}\left[-\frac{N}{2}+1\right], \ldots, w_{(n)}\left[\frac{N}{2}-1\right]\right]^{T}$ is a white complex Gaussian noise vector of covariance matrix $N_{T} \cdot \sigma^{2} \mathbf{I}_{N}$ and $\mathbf{H}_{(n)}$ is a $N_{R} N \times N_{T} N$ MIMO channel matrix given by:

$$
\mathbf{H}_{(n)}=\left[\begin{array}{ccc}
\mathbf{H}_{(1,1, n)} & \cdots & \mathbf{H}_{\left(1, N_{T}, n\right)} \\
\vdots & \ddots & \vdots \\
\mathbf{H}_{\left(N_{R}, 1, n\right)} & \cdots & \mathbf{H}_{\left(N_{R}, N_{T}, n\right)}
\end{array}\right]
$$

where $\mathbf{H}_{(r, t, n)}$ is the channel matrix between the $t$ th transmit antenna and the $r$ th receive antenna. The elements of channel matrix $\mathbf{H}_{(r, t, n)}$ can be written in terms of equivalent channel taps [2] $\left\{g_{(l, r, t)}^{(n)}\left(q T_{s}\right)=g_{(l, r, t)}\left(n T+q T_{s}\right)\right\}$ or in terms of physical channel parameters [8]: delays $\left\{\tau_{(l, r, t)}\right\}$ and complex gains $\left\{\alpha_{(l, r, t)}^{(n)}\left(q T_{s}\right)=\alpha_{(l, r, t)}\left(n T+q T_{s}\right)\right\}$, as:

$$
\begin{gathered}
{\left[\mathbf{H}_{(r, t, n)}\right]_{k, m}} \\
=\frac{1}{N} \sum_{l=1}^{L_{(r, t)}^{\prime}}\left[e^{-j 2 \pi\left(\frac{m-1}{N}-\frac{1}{2}\right)(l-1)} \sum_{q=0}^{N-1} g_{(l, r, t)}^{(n)}\left(q T_{s}\right) e^{j 2 \pi \frac{m-k}{N} q}\right] \\
=\frac{1}{N} \sum_{l=1}^{L_{(r, t)}}\left[e^{-j 2 \pi\left(\frac{m-1}{N}-\frac{1}{2}\right) \tau(l, r, t)} \sum_{q=0}^{N-1} \alpha_{(l, r, t)}^{(n)}\left(q T_{s}\right) e^{j 2 \pi \frac{m-k}{N} q}\right](4)
\end{gathered}
$$

where $L_{(r, t)}^{\prime}<N_{g}$ and $L_{(r, t)}$ are respectively the number of channel taps and the number of paths for the branch between the $t$ th transmit antenna and the $r$ th receive antenna. The delays are normalized by $T_{s}$ and not necessarily integers $\left(\tau_{(l, r, t)}<N_{g}\right)$. The $L_{(r, t)}$ elements of $\left\{\alpha_{(l, r, t)}^{(n)}\left(q T_{s}\right)\right\}$ are uncorrellated. Howerver, the $L_{(r, t)}^{\prime}$ elements of $\left\{g_{(l, r, t)}^{(n)}\left(q T_{s}\right)\right\}$ are correlated, unless that the delays are multiple of $T_{s}$ as mostly assumed in the litterature. They are wide-sense stationary (WSS), narrow-band zero-mean complex Gaussian processes of variances $\sigma_{g_{(l, r, t)}}^{2}$ and $\sigma_{\alpha_{(l, r, t)}}^{2}$, with the socalled Jakes' power spectrum of maximum Doppler frequency $f_{d}$ [18]. The average energy of each channel approach is normalized to one, i.e., $\sum_{l=1}^{L_{(r, t)}^{\prime}} \sigma_{g_{(l, r, t)}}^{2}=1$ and $\sum_{l=1}^{L_{(r, t)}} \sigma_{\alpha_{(l, r, t)}}^{2}=1$.

In the sequel, we will make the calculus based on the second approach (physical channel) and we can deduce the results of the first approach (channel taps) by replacing $L_{(r, t)}$ by $L_{(r, t)}^{\prime}$ and the set of delays $\left\{\tau_{(l, r, t)}\right\}$ by $\left\{l-1, l=1: L_{(r, t)}^{\prime}\right\}$.

\section{B. BEM Channel Model}

Let $L=\sum_{r=1}^{N_{R}} L_{(r)}=\sum_{r=1}^{N_{R}} \sum_{t=1}^{N_{T}} L_{(r, t)}$ be the total number of complex gains for the MIMO channel and designate by $(t, r)$ the branch between the $t$ th transmit antenna and the $r$ th receive antenna. Since the number of samples to estimate $L v$ is greater than the number of observation equations $N_{R} N$, it is not efficient to estimate the time-variation of the complex gains, using directly the observation model in (1). Thus, we need to reduce the number of parameters to estimate. In this section, our aim is to accurately model the time-variation of $\alpha_{(l, r, t)}^{(n)}\left(q T_{s}\right)$ by using a BEM. Collecting the samples of the $l$ th path of the branch $(r, t)$ within the $n$th MIMO-OFDM block in a $v \times 1$ vector $\boldsymbol{\alpha}_{(l, r, t)}^{(n)}=\left[\alpha_{(l, r, t)}^{(n)}\left(-N_{g} T_{s}\right), \ldots, \alpha_{(l, r, t)}^{(n)}\left((N-1) T_{s}\right)\right]^{T}$, we can express $\boldsymbol{\alpha}_{(l, r, t)}^{(n)}$ as:

$\boldsymbol{\alpha}_{(l, r, t)}^{(n)}=\boldsymbol{\alpha}_{\mathbf{B E M}_{(l, r, t)}^{(n)}}+\boldsymbol{\xi}_{(l, r, t)}^{(n)}=\mathbf{Q} \mathbf{c}_{(l, r, t)}^{(n)}+\boldsymbol{\xi}_{(l, r, t)}^{(n)}$

where $\mathbf{Q}=\left[\mathbf{q}_{1}, \ldots, \mathbf{q}_{N_{c}}\right]$ is a $v \times N_{c}$ matrix that collects $N_{c}$ orthonormal basis function $\mathbf{q}_{d}$ as columns, $\mathbf{c}_{(l, r, t)}^{(n)}=$ $\left[c_{(1, l, r, t)}^{(n)}, \ldots, c_{\left(N_{c}, l, r, t\right)}^{(n)}\right]^{T}$ represent the $N_{c}$ BEM coefficients for the $l$ th complex gain of the branch $(r, t)$ of the $n$th MIMOOFDM block, and $\boldsymbol{\xi}_{(l, r, t)}^{(n)}$ represents the corresponding BEM modeling error, which is assumed to be minimized in the MSE sense [16] [5]. Under this criterion, the optimal BEM coefficients and the corresponding model error are given by:

$$
\begin{aligned}
& \mathbf{c}_{(l, r, t)}^{(n)}=\left(\mathbf{Q}^{H} \mathbf{Q}\right)^{-1} \mathbf{Q}^{H} \boldsymbol{\alpha}_{(l, r, t)}^{(n)} \\
& \boldsymbol{\xi}_{(l, r, t)}^{(n)}=\left(\mathbf{I}_{v}-\mathbf{S}\right) \boldsymbol{\alpha}_{(l, r, t)}^{(n)}
\end{aligned}
$$

where $\mathbf{S}=\mathbf{Q}\left(\mathbf{Q}^{H} \mathbf{Q}\right)^{-1} \mathbf{Q}^{H}$ is a $v \times v$ matrix. It provides the MMSE approximation for all BEM containing $N_{c}$ coefficients, given by:

$$
\begin{aligned}
\operatorname{MMSE}_{(l, r, t)} & =\frac{1}{v} \mathrm{E}\left[\boldsymbol{\xi}_{(l, r, t)}^{(n)} \boldsymbol{\xi}_{(l, r, t)}^{(n)}{ }^{H}\right] \\
& =\frac{1}{v} \operatorname{Tr}\left(\left(\mathbf{I}_{v}-\mathbf{S}\right) \mathbf{R}_{\boldsymbol{\alpha}_{(l, r, t)}^{(0)}}\left(\mathbf{I}_{v}-\mathbf{S}^{H}\right)\right)
\end{aligned}
$$

where $\mathbf{R}_{\boldsymbol{\alpha}_{(l, r, t)}^{(s)}}^{(s)}=\mathrm{E}\left[\boldsymbol{\alpha}_{(l, r, t)}^{(n)} \boldsymbol{\alpha}_{(l, r, t)}^{(n-s)^{H}}\right]$ is the $v \times v$ correlation matrix of $\boldsymbol{\alpha}_{(l, r, t)}^{(n)}$ with elements given by:

$$
\left[\mathbf{R}_{\boldsymbol{\alpha}_{(l, r, t)}}^{(s)}\right]_{k, m}=\sigma_{\alpha_{(l, r, t)}}^{2} J_{0}\left(2 \pi f_{d} T_{s}(k-m+s v)\right)
$$

Various traditional BEM designs have been reported to model the channels time-variations, e.g., the CE-BEM $[\mathbf{Q}]_{k, m}=$ $e^{j 2 \pi\left(\frac{k-N g-1}{v}\right)\left(m-1-\frac{N_{c}-1}{2}\right)}$ [15], the GCE-BEM $[\mathbf{Q}]_{k, m}=$ 
$e^{j 2 \pi\left(\frac{k-N g-1}{a v}\right)\left(m-1-\frac{N_{c}-1}{2}\right)}$ with $1<a \leq \frac{N_{c}-1}{2 f_{d} T}$ [16], the P$\mathrm{BEM}[\mathbf{Q}]_{k, m}=(k-N g-1)^{(m-1)}$ [5] and the DKL-BEM which employs basis sequences that corresponds to the most significant eigenvectors of the autocorrelation matrix $\mathbf{R}_{\boldsymbol{\alpha}_{(l, r, t)}^{(0)}}$ [17]. From now on, we can describe the MIMO-OFDM system model derived previously in terms of the BEM. Substituting (5) in (1) and neglecting the BEM model error, we obtain after some algebra:

$$
\mathbf{y}_{(n)}=\mathcal{K}_{(n)} \mathbf{c}_{(n)}+\mathbf{w}_{(n)}
$$

where the $L N_{c} \times 1$ vector $\mathbf{c}_{(n)}$ and the $N_{R} N \times L N_{c}$ matrix $\mathcal{K}_{(n)}$ are given by:

$$
\begin{aligned}
\mathbf{c}_{(n)} & =\left[\mathbf{c}_{(1,1, n)}^{T}, \ldots, \mathbf{c}_{\left(1, N_{T}, n\right)}^{T}, \ldots, \mathbf{c}_{\left(N_{R}, N_{T}, n\right)}^{T}\right]^{T} \\
\mathbf{c}_{(r, t, n)} & =\left[\mathbf{c}_{(1, r, t)}^{(n)^{T}}, \ldots, \mathbf{c}_{\left(L_{(r, t)}, r, t\right)}^{(n)^{T}}\right]^{T} \\
\mathcal{K}_{(n)} & =\operatorname{blkdiag}\left\{\mathcal{K}_{(1, n)}, \ldots, \mathcal{K}_{\left(N_{R}, n\right)}\right\} \\
\mathcal{K}_{(r, n)} & =\left[\mathcal{K}_{(r, 1, n)}, \ldots, \mathcal{K}_{\left(r, N_{T}, n\right)}\right] \\
\mathcal{K}_{(r, t, n)} & =\frac{1}{N}\left[\mathbf{Z}_{(1, r, t)}^{(n)}, \ldots, \mathbf{Z}_{\left(L_{r, t)}, r, t\right)}^{(n)}\right] \\
\mathbf{Z}_{(l, r, t)}^{(n)} & =\left[\mathbf{M}_{(1)} \operatorname{diag}\left\{\mathbf{x}_{(t, n)}\right\} \mathbf{f}_{(l, r, t)}, \ldots, \mathbf{M}_{\left(N_{c}\right)} \operatorname{diag}\left\{\mathbf{x}_{(t, n)}\right\} \mathbf{f}_{(l, r, t)}\right]
\end{aligned}
$$

where $\mathbf{f}_{(l, r, t)}$ is the $l$ th column of the $N \times L_{(r, t)}$ Fourier matrix $\mathbf{F}_{(r, t)}$ and $\mathbf{M}_{(d)}$ is a $N \times N$ matrix given by:

$$
\begin{aligned}
{\left[\mathbf{F}_{(r, t)}\right]_{k, l} } & =e^{-j 2 \pi\left(\frac{k-1}{N}-\frac{1}{2}\right) \tau_{(l, r, t)}} \\
{\left[\mathbf{M}_{(d)}\right]_{k, m} } & =\sum_{q=0}^{N-1}[\mathbf{Q}]_{q+N_{g}+1, d} e^{j 2 \pi \frac{m-k}{N} q}
\end{aligned}
$$

Moreover, the channel matrix of the branch $(r, t)$ can be easily computed by using the BEM coefficients [9]:

$$
\mathbf{H}_{(r, t, n)}=\sum_{d=1}^{N_{c}} \mathbf{M}_{(d)} \operatorname{diag}\left\{\mathbf{F}_{(r, t)} \chi_{(d, r, t)}^{(n)}\right\}
$$

where $\chi_{(d, r, t)}^{(n)}=\left[c_{(d, 1, r, t)}^{(n)}, \ldots, c_{\left(d, L_{(r, t,)}, r, t\right)}^{(n)}\right]^{T}$. The matrices $\mathbf{M}_{(d)}$ can be computed and stored.

\section{AR Model AND Kalman Filter}

\section{A. The AR Model for $\boldsymbol{c}^{(n)}$}

The optimal BEM coefficients $\mathbf{c}_{(l, r, t)}^{(n)}$ are correlated complex Gaussian variables with zero-means and correlation matrix given by:

$$
\begin{aligned}
\mathbf{R}_{\mathbf{c}_{(l, r, t)}^{(s)}}^{(s)} & =\mathrm{E}\left[\mathbf{c}_{(l, r, t)}^{(n)} \mathbf{c}_{(l, r, t)}^{(n-s)}{ }^{H}\right] \\
& =\left(\mathbf{Q}^{H} \mathbf{Q}\right)^{-1} \mathbf{Q}^{H} \mathbf{R}_{\boldsymbol{\alpha}_{(l, r, t)}}^{(s)} \mathbf{Q}\left(\mathbf{Q}^{H} \mathbf{Q}\right)^{-1}
\end{aligned}
$$

Hence, the dynamics of $\mathbf{c}_{(l, r, t)}^{(n)}$ can be well modeled by an auto-regressive (AR) process [19] [20] [8] . A complex AR process of order $p$ can be generated as:

$$
\mathbf{c}_{(l, r, t)}^{(n)}=-\sum_{i=1}^{p} \mathbf{A}^{(i)} \mathbf{c}_{(l, r, t)}^{(n-i)}+\mathbf{u}_{(l, r, t)}^{(n)}
$$

where $\mathbf{A}^{(1)}, \ldots, \mathbf{A}^{(p)}$ are $N_{c} \times N_{c}$ matrices and $\mathbf{u}_{(l, r, t)}^{(n)}$ is a $N_{c} \times 1$ complex Gaussian vector with covariance matrix
$\mathbf{U}_{(l, r, t)}$. The matrices $\mathbf{A}^{(1)}, \ldots, \mathbf{A}^{(p)}$ and $\mathbf{U}_{(l, r, t)}$ are the AR model parameters obtained by solving the set of Yule-Walker equations defined as:

$\mathbf{T A}=-\mathbf{V} \quad$ and $\quad \mathbf{U}_{(l, r, t)}=\mathbf{R}_{\mathbf{c}_{(l, r, t)}^{(0)}}+\sum_{i=1}^{p} \mathbf{A}^{(i)} \mathbf{R}_{\mathbf{c}_{(l, r, t)}^{(-i)}}^{(1)}$

where $\mathbf{A}=\left[\mathbf{A}^{(1)^{T}}, \ldots, \mathbf{A}^{(p)^{T}}\right]^{T}$ is $p N_{c} \times N_{c}$ matrix, $\mathbf{V}$ and $\mathbf{T}$ are $p N_{c} \times N_{c}$ and $p N_{c} \times p N_{c}$ matrices defined by:

$$
\begin{aligned}
& \mathbf{V}=\frac{1}{\sigma_{\alpha_{(l, r, t)}}^{2}}\left[\mathbf{R}_{\mathbf{c}_{(l, r, t)}^{(1)^{T}}, \ldots, \mathbf{R}_{\mathbf{c}_{(l, r, t)}}^{(p)^{T}}}^{(1}\right]^{T} \\
& \mathbf{T}=\frac{1}{\sigma_{\alpha_{(l, r, t)}}^{2}}\left[\begin{array}{ccc}
\mathbf{R}_{\mathbf{c}_{(l, r, t)}^{(0)}}^{(0)} & \cdots & \mathbf{R}_{\mathbf{c}_{(l, r, t)}^{(-p+1)}} \\
\vdots & \ddots & \vdots \\
\mathbf{R}_{\mathbf{c}_{(l, r, t)}^{(p-1)}}^{(p-1)} & \cdots & \mathbf{R}_{\mathbf{c}_{(l, r, t)}^{(0)}}^{(0)}
\end{array}\right]
\end{aligned}
$$

Using (16), we obtain the AR model of order $p$ for $\mathbf{c}_{(n)}$ :

$$
\mathbf{c}_{(n)}=-\sum_{i=1}^{p} \mathcal{A}_{(i)} \mathbf{c}_{(n-i)}+\mathbf{u}_{(n)}
$$

where $\mathcal{A}_{(i)}=\operatorname{blkdiag}\left\{\mathbf{A}^{(i)}, \ldots, \mathbf{A}^{(i)}\right\}$ is a $L N_{c} \times L N_{c}$ matrix and $\mathbf{u}_{(n)}=\left[\mathbf{u}_{(1,1,1)}^{(n)^{T}}, \ldots, \mathbf{u}_{\left(L_{\left(N_{R}, N_{T}\right)}, N_{R}, N_{T}\right)}^{(n)^{T}}\right]^{T}$ is a $L N_{c} \times 1$ complex Gaussian vector with covariance matrix $\mathbf{U}=\operatorname{blkdiag}\left\{\mathbf{U}_{(1,1,1)}, \ldots, \mathbf{U}_{\left(L_{\left(N_{R}, N_{T}\right)}, N_{R}, N_{T}\right)}\right\}$.

\section{B. The Kalman Filter}

Based on the AR model of $\mathbf{c}_{(n)}$ in (20), we define the state space model for the MIMO-OFDM system as $\mathbf{g}_{(n)}=$ $\left[\mathbf{c}_{(n)}^{T}, \ldots, \mathbf{c}_{(n-p+1)}^{T}\right]^{T}$. Thus, using (20) and (11), we obtain:

$$
\begin{aligned}
& \mathbf{g}_{(n)}=\mathbf{S}_{1} \mathbf{g}_{(n-1)}+\mathbf{S}_{2} \mathbf{u}_{(n)} \\
& \mathbf{y}_{(n)}=\mathbf{S}_{3} \mathbf{g}_{(n)}+\mathbf{w}_{(n)}
\end{aligned}
$$

where $\mathbf{S}_{2}=\left[\mathbf{I}_{L N_{c}}, \mathbf{0}_{L N_{c},(p-1) L N_{c}}\right]^{T}$ is a $p L N_{c} \times L N_{c}$ matrix, $\mathbf{S}_{3}=\left[\mathcal{K}_{(n)}, \mathbf{0}_{N,(p-1) L N_{c}}\right]$ is a $N \times p L N_{c}$ matrix and $\mathbf{S}_{1}$ is a $p L N_{c} \times p L N_{c}$ matrix defined as:

$$
\mathbf{S}_{1}=\left[\begin{array}{ccccc}
-\mathcal{A}_{(1)} & -\mathcal{A}_{(2)} & -\mathcal{A}_{(3)} & \ldots & -\mathcal{A}_{(p)} \\
\mathbf{I}_{L N_{c}} & \mathbf{0}_{L N_{c}} & \mathbf{0}_{L N_{c}} & \ldots & \mathbf{0}_{L N_{c}} \\
\mathbf{0}_{L N_{c}} & \mathbf{I}_{L N_{c}} & \mathbf{0}_{L N_{c}} & \ldots & \mathbf{0}_{L N_{c}} \\
\vdots & \ddots & \ddots & \ddots & \vdots \\
\mathbf{0}_{L N_{c}} & \cdots & \mathbf{0}_{L N_{c}} & \mathbf{I}_{L N_{c}} & \mathbf{0}_{L N_{c}}
\end{array}\right]
$$

The state and the observation models (21) (22) allow us to use Kalman filter to adaptively track the BEM coefficients $\mathbf{c}_{(n)}$. Let $\hat{\mathbf{g}}_{(n)}$ be our a priori state estimate at step $n$ given knowledge of the process prior to step $n, \hat{\mathbf{g}}_{(n \mid n)}$ be our a posteriori state estimate at step $n$ given measurement $\mathbf{y}_{(n)}$ and, $\mathbf{P}_{(n)}$ and $\mathbf{P}_{(n \mid n)}$ are the a priori and the a posteriori error estimate covariance matrix of size $p L N_{c} \times p L N_{c}$, respectively. We initialize the Kalman filter with $\mathbf{g}_{(0 \mid 0)}=\mathbf{0}_{p L N_{c}, 1}$ and $\mathbf{P}_{(0 \mid 0)}$ given by:

$$
\begin{aligned}
& \mathbf{P}_{(0 \mid 0)\left[u(s), u\left(s^{\prime}\right)\right]}=\mathbf{R}_{\mathbf{c}}^{\left(s-s^{\prime}\right)}
\end{aligned}
$$

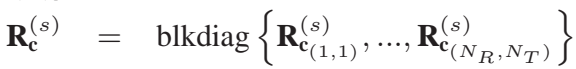

$$
\begin{aligned}
& \mathbf{R}_{\mathbf{c}_{(r, t)}}^{(s)}=\operatorname{blkdiag}\left\{\mathbf{R}_{\mathbf{c}_{(1, r, t)}}^{(s)}, \ldots, \mathbf{R}_{\left.\mathbf{c}_{(L}(r, t), r, t\right)}^{(s)}\right\}
\end{aligned}
$$


where $u(s)=1+L N_{c}:(s+1) L N_{c}$ with $s \in[0, p-1]$ and $\mathbf{R}_{\mathbf{c}_{(l, r, t)}^{(s)}}^{(i)}$ is the correlation matrix of $\mathbf{c}_{(l, r, t)}^{(n)}$ defined in (15). The Kalman filter is a recursive algorithm composed of two stages: Time Update Equations and Measurement Update Equations. These two stages are defined as:

\section{Time Update Equations:}

$$
\begin{aligned}
\hat{\mathbf{g}}_{(n)} & =\mathbf{S}_{1} \hat{\mathbf{g}}_{(n-1 \mid n-1)} \\
\mathbf{P}_{(n)} & =\mathbf{S}_{1} \mathbf{P}_{(n-1 \mid n-1)} \mathbf{S}_{1}^{H}+\mathbf{S}_{2} \mathbf{U} \mathbf{S}_{2}^{H}
\end{aligned}
$$

\section{Measurement Update Equations:}

$$
\begin{aligned}
\mathbf{K}_{(n)} & =\mathbf{P}_{(n)} \mathbf{S}_{3}^{H}\left(\mathbf{S}_{3} \mathbf{P}_{(n)} \mathbf{S}_{3}^{H}+N_{T} \cdot \sigma^{2} \mathbf{I}_{N_{R} N}\right)^{-1} \\
\hat{\mathbf{g}}_{(n \mid n)} & =\hat{\mathbf{g}}_{(n)}+\mathbf{K}_{(n)}\left(\mathbf{y}_{(n)}-\mathbf{S}_{3} \hat{\mathbf{g}}_{(n)}\right) \\
\mathbf{P}_{(n \mid n)} & =\mathbf{P}_{(n)}-\mathbf{K}_{(n)} \mathbf{S}_{3} \mathbf{P}_{(n)}
\end{aligned}
$$

where $\mathbf{K}_{(n)}$ is the Kalman gain. The Time Update Equations are responsible for projecting forward (in time) the current state and error covariance estimates to obtain the a priori estimates for the next time step. The Measurement Update Equations are responsible for the feedback, i.e., for incorporating a new measurement into the a priori estimate to obtain an improved a posteriori estimate. The Time Update Equations can also be thought of a predictor equations, while the Measurement Update Equations can be thought of a corrector equations.

\section{Joint Data Detection And Kalman Estimation}

\section{A. Iterative Algorithm}

In the iterative algorithm for joint data detection and channel Kalman estimation, the $N_{p}$ pilots subcarriers are evently inserted into the $\mathrm{N}$ subcarriers at the positions $\mathcal{P}=\left\{p_{r} \mid p_{r}=\right.$ $\left.(r-1) L_{f}+1, r=1, \ldots, N_{p}\right\}$, where $L_{f}$ is the distance between two adjacent pilots. We test two channel equalizers for the data detection: the MMSE equalizer [13] and the QRequalizer [8] [7]. The first one is based on the noise variance $N_{T} . \sigma^{2}$ whereas the second equalizer allows us to estimate the data symbol with free ICI by performing a so-called QRdecomposition. The algorithm proceeds as follows:

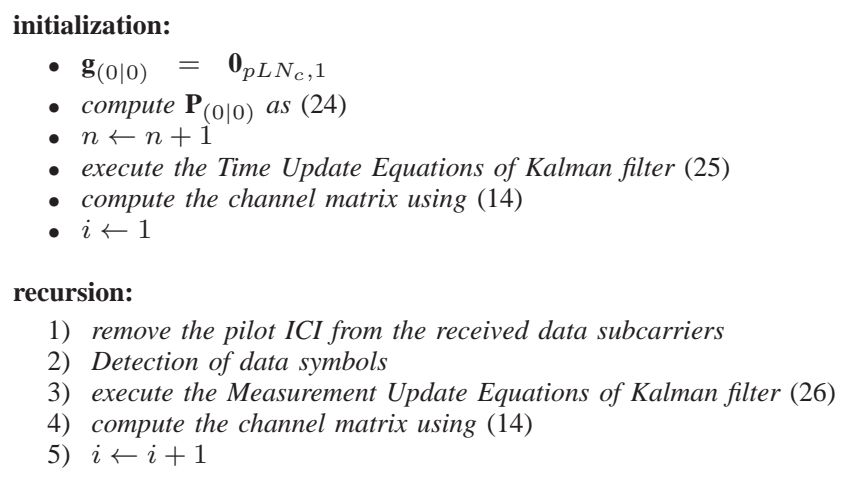

where $i$ represents the iteration number.

\section{B. Mean Square Error (MSE) Analysis}

The error between the $l$ th exact complex gain and the $l$ th estimated polynomial $\hat{\boldsymbol{\alpha}}_{\mathbf{B E M}}^{(n)} \boldsymbol{M}_{(l, r, t)}$ is given by:

$\mathbf{e}_{(l, r, t)}^{(n)}=\boldsymbol{\alpha}_{(l, r, t)}^{(n)}-\hat{\boldsymbol{\alpha}}_{\mathbf{B E M}(l, r, t)}^{(n)}=\boldsymbol{\xi}_{(l, r, t)}^{(n)}+\mathbf{Q} \mathbf{e}_{(l, r, t)}^{(n)}$

where $\mathbf{e}_{\mathbf{c}_{(l, r, t)}^{(n)}}^{(n)}=\mathbf{c}_{(l, r, t)}^{(n)}-\hat{\mathbf{c}}_{(l, r, t)}^{(n)}$ and $\boldsymbol{\xi}_{(l, r, t)}^{(n)}$ is the BEM model error defined in (7). Neglecting the cross-covariance terms between $\boldsymbol{\xi}_{(l, r, t)}^{(n)}$ and $\mathbf{e}_{\mathbf{c}_{(l, r, t)}^{(n)}}^{(n)}$, the mean square error (MSE) between $\boldsymbol{\alpha}_{(l, r, t)}^{(n)}$ and $\boldsymbol{\alpha}_{\mathbf{B E M}}^{(n)} \mathbf{M}_{(l, r, t)}$ is given by:

$$
\begin{aligned}
\operatorname{MSE}_{(l, r, t)} & =\frac{1}{v} \mathrm{E}\left[\mathbf{e}_{(l, r, t)}^{(n)^{H}} \mathbf{e}_{(l, r, t)}^{(n)}\right] \\
& =\operatorname{MMSE}_{(l, r, t)}+\frac{1}{v} \operatorname{Tr}\left(\mathbf{Q} \mathbf{M S E}_{\mathbf{c}_{(l, r, t)}} \mathbf{Q}^{H}\right)
\end{aligned}
$$

where $\mathbf{M S E}_{\mathbf{c}_{(l, r, t)}}=\mathrm{E}\left[\mathbf{e}_{\mathbf{c}_{(l, r, t)}^{(n)}}^{(n)} \mathbf{e}_{\mathbf{c}_{(l, r, t)}^{(n)}}^{(n)}{ }^{H}\right]$. Notice that, at the convergence of the Kalman filter, we have:

$$
\operatorname{MSE}_{\mathbf{c}_{(l, r, t)}}=\mathbf{P}_{(n \mid n)[f(l, r, t), f(l, r, t)]}
$$

provided that the data symbols are perfectly estimated (i.e., data-aided context), where the sequence of indices $f(l, r, t)=1+\left(l-1+a_{(r, t)}\right) N_{c}:\left(l+a_{(r, t)}\right) N_{c}$ with $a_{(r, t)}=\sum_{r^{\prime}=1}^{r-1} \sum_{t^{\prime}=1}^{t-1} L_{\left(r^{\prime}, t^{\prime}\right)}$

The on-line Bayesian Cramer-Rao Bound (BCRB) is an important criterion for evaluting the quality of the complex gains Kalman estimation. The on-line BCRB for the estimation of $\boldsymbol{\alpha}_{(l, r, t)}^{(n)}$, in data-aided (DA) context, is given by:

$\operatorname{BCRB}\left(\boldsymbol{\alpha}_{(l, r, t)}^{(\infty)}\right)=\operatorname{MMSE}_{(l, r, t)}+\frac{1}{v} \operatorname{Tr}\left(\mathbf{Q B C R B}\left(\mathbf{c}_{(l, r, t)}^{(\infty)}\right) \mathbf{Q}^{H}\right)$

where $\mathbf{B C R B}\left(\mathbf{c}_{(l, r, t)}^{(K)}\right)$ is the on-line BCRB associated to the estimation of $\mathbf{c}_{(l, r, t)}^{(K)}$ which is given by:

$$
\operatorname{BCRB}\left(\mathbf{c}_{(l, r, t)}^{(K)}\right)=\operatorname{BCRB}(\mathbf{c})_{[f(l, r, t), f(l, r, t)]}
$$

$\operatorname{BCRB}(\mathbf{c})$ is the on-line BCRB for the estimation of $\mathbf{c}=$ $\left[\mathbf{c}_{(K)}^{T}, \ldots, \mathbf{c}_{(1)}^{T}\right]^{T}$ in DA context which is given by:

$$
\mathbf{B C R B}(\mathbf{c})=\left(\operatorname{blkdiag}\left\{\mathbf{J}_{(K)}, \ldots, \mathbf{J}_{(2)}, \mathbf{J}_{(1)}\right\}+\mathbf{R}_{\mathbf{c}}^{-1}\right)^{-1}
$$

where $\mathbf{R}_{\mathbf{c}}$ is calculated in the same way as $\mathbf{P}_{(0 \mid 0)}$ with $s, s^{\prime} \in$ $[0, K-1]$, and $\mathbf{J}_{(n)}$ is a $L N_{c} \times L N_{c}$ matrix given by [5]:

$$
\begin{aligned}
& \mathbf{J}_{(n)}=\operatorname{blkdiag}\left\{\mathbf{J}_{(1, n)}, \ldots, \mathbf{J}_{\left(N_{R}, n\right)}\right\} \\
& \mathbf{J}_{(r, n)}=\left[\begin{array}{ccc}
\mathbf{J}_{(r, 1,1, n)} & \cdots & \mathbf{J}_{\left(r, 1, N_{T}, n\right)} \\
\vdots & \ddots & \vdots \\
\mathbf{J}_{\left(r, N_{T}, 1, n\right)} & \cdots & \mathbf{J}_{\left(r, N_{T}, N_{T}, n\right)}
\end{array}\right] \\
& \mathbf{J}_{\left(r, t, t^{\prime}, n\right)}=\frac{1}{N^{2} N_{T} \sigma^{2}} \mathcal{F}_{(r, t, n)}^{H} \\
& \mathcal{M} \mathcal{F}_{\left(r, t^{\prime}, n\right)}
\end{aligned}
$$




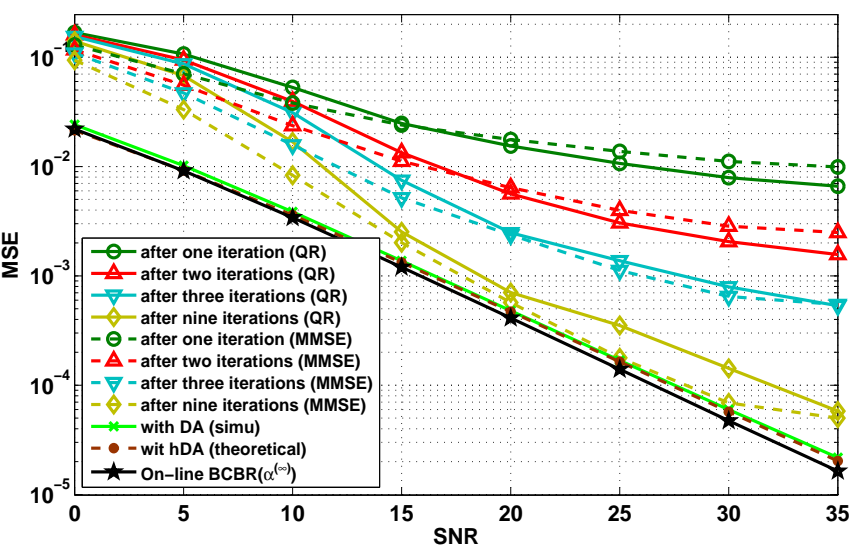

Fig. 1. MSE vs SNR for $f_{d} T=0.5$ and GCE-BEM with $N_{c}=4$

where $\mathcal{M}$ and $\mathcal{F}_{(r, t, n)}$ are a $N N_{c} \times N N_{c}$ and a $N N_{c} \times$ $L_{(r, t)} N_{c}$ matrices, respectively, defined as:

$$
\begin{aligned}
\mathcal{M} & =\left[\begin{array}{ccc}
\mathbf{M}_{(1)}^{H} \mathbf{M}_{(1)} & \cdots & \mathbf{M}_{(1)}^{H} \mathbf{M}_{\left(N_{c}\right)} \\
\vdots & \ddots & \vdots \\
\mathbf{M}_{\left(N_{c}\right)}^{H} \mathbf{M}_{(1)} & \cdots & \mathbf{M}_{\left(N_{c}\right)}^{H} \mathbf{M}_{\left(N_{c}\right)}
\end{array}\right] \\
\mathcal{F}_{(r, t, n)} & =\left[\begin{array}{lll}
\mathcal{F}_{(1, r, t, n)} & \cdots & \mathcal{F}_{\left(L_{(r, t)}, r, t, n\right)}
\end{array}\right]
\end{aligned}
$$

where the $N N_{c} \times N_{c}$ matrix $\mathcal{F}_{(l, r, t, n)}$ is given by:

$$
\begin{aligned}
& \mathcal{F}_{(l, r, t, n)}= \\
& \text { blkdiag }\left\{\operatorname{diag}\left\{\mathbf{x}_{(t, n)}\right\} \mathbf{f}_{(l, r, t)}, \ldots, \operatorname{diag}\left\{\mathbf{x}_{(t, n)}\right\} \mathbf{f}_{(l, r, t)}\right\}
\end{aligned}
$$

\section{Simulation}

In this section, we verify the theory by simulation and we test the performance of the iterative algorithm based on the second approach (i.e., estimation of $\boldsymbol{\alpha}$ assuming the availability of delay information). We assume that the channels from different transmitters to the different receivers have the same delay and fading property (i.e., same number of paths, $\sigma_{\alpha_{(l, r, t)}}^{2}$ and $\left.\tau_{(l, r, t)}\right)$ and we use the Rayleigh channel model given in [8] [5]. It is reasonable because the transmitters and the receivers are very close to each other in practice. A normalized 4QAM MIMO-OFDM system, with two transmit and two receive antennas, $N=256$ subcarriers, $N_{g}=\frac{N}{8}$, $N_{p}=\frac{N}{4}$ pilots (i.e., $L_{f}=4$ ) and $\frac{1}{T_{s}}=2 M H z$ is used (note that SNR $=\frac{1}{\sigma^{2}}$ ). The MSE and the BER are evaluated under a rapid time-varying channel such as $f_{d} T=0.5$ corresponding to a vehicle speed $V_{m}=400 \mathrm{~km} / \mathrm{h}$ for $f_{c}=10 \mathrm{GHz}$. In order to decrease the complexity of the Kalman filter, we choose an AR model of order $p=1$.

Fig. 1 and Fig. 2 show respectively the evolution of MSE and BER versus SNR, with the iterations, for $f_{d} T=0.5$ and GCE-BEM. We select $N_{c}=4$ in order to reduce the impact of the BEM modeling error. The equalizers used for data detection are: the MMSE equalizer [13] and the QRequalizer [8] [7]. From Fig. 1, it is shown that, with DA, the MSE obtained by simulation agrees with the theoretical value of MSE given by (29). We also observe that MSE with $\mathrm{DA}$ is very close to the on-line BCRB. This means that the Kalman filter works very well. After three and nine iterations, a great improvement is realized and the MSE is close to the

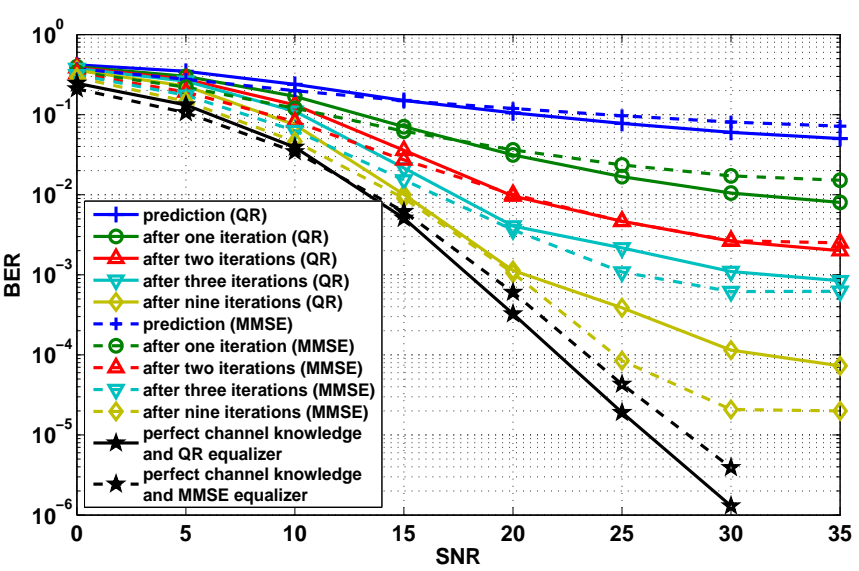

Fig. 2. BER vs SNR for $f_{d} T=0.5$ and GCE-BEM with $N_{c}=4$

MSE with DA, particularly in regions of moderate and high SNR. In Fig. 2, we plotted, as reference, the performance obtained with perfect channel knowledge. After three and nine iterations, a significant improvement occurs; the performance of our algorithm and the performance obtained with perfect channel knowledge are very close, particularly in regions of low and moderate SNR. At very high SNR, it is normal to not reach the reference because we have a small error floor due to the data symbol detection error. It sould be noted that the error floor with QR-equalizer is larger than that of the MMSE equalizer.

\section{CONCLUSION}

In this paper, we have presented a new iterative algorithm for joint multi-path Rayleigh channel estimation and data recovery in fast time-varying environments. The rapid timevariation of the channel within one MIMO-OFDM block are approximated by a BEM model. The BEM coefficients are tracked and estimated using the Kalman filter. The data symbols are estimated by performing a QR-decomposition of the channel matrix or by using a MMSE equalizer. Theoretical analysis and simulation results show that our algorithm has a good performance for high Doppler spread.

\section{ACKOWLEDGEMENT}

This work has been carried out in the framework of the CISIT (Campus International sur la Sécurité et Intermodalité des Transports) project and funded by the French Ministry of Research, the Region Nord Pas de Calais and the European Commission (FEDER funds)

\section{REFERENCES}

[1] Z. J. Wang and Z. Han, "A MIMO-OFDM Channel Estimation Approach Using Time of Arrivals" in IEEE Trans. Wirel. Comm., vol. 4. no. 3, May 2005.

[2] J.-G KIM and J.-T LIM, "MAP-Based Channel Estimation for MIMOOFDM Over Fast Rayleigh Fading Channels" in IEEE Trans. Vehic. Techno., vol. 57. no. 3, May 2008.

[3] Y. Li, " Simplified Channel Estimation for OFDM Systems with Multiple Transmit Antennas" in IEEE Trans. Wireless Comm., vol. 1. no. 1, Jan. 2002.

[4] Z. Jane Wang, Zhu Han, and K. J. Ray Liu,“ MIMO-OFDM Channel Estimation via Probabilistic Data Association Based TOAs" in IEEE GLOBAL COMMUNICATIONS Conf., pp. 626-630, Dec. 2003. 
[5] H. Hijazi and L. Ros, " Analytical Analysis of Bayesian Cramer-Rao Bound for Dynamical Rayleigh Channel Complex Gains Estimation in OFDM System ” IEEE Trans. Signal Process., vol. 57, No. 5, May 2009.

[6] H. Hijazi and L. Ros, "OFDM High Speed Channel Complex Gains Estimation Using Kalman Filter and QR-Detector" in IEEE ISWCS Conf., Reykjavik, Iceland, October 2008.

[7] H. Hijazi and L. Ros, “Joint Data QR-Detection and Kalman Estimation for OFDM Time-varying Rayleigh Channel Complex Gains" in IEEE Trans. Comm., appeared in 2010.

[8] H. Hijazi and L. Ros, " Polynomial Estimation of Time-varying Multipath Gains with Intercarrier Interference Mitigation in OFDM Systems" in IEEE Trans. Vehic. Techno., Vol. 57, No. 6, November 2008.

[9] H. Hijazi and L. Ros, " Rayleigh Time-varying Channel Complex Gains Estimation and ICI Cancellation in OFDM Systems" in European Transactions on Telecommunications, June 2009.

[10] E. Simon, L. Ros and K. Raoof,“ Synchronization over Rapidly Timevarying Multipath Channel for CDMA Downlink RAKE Receivers in Time-Division Mode" in IEEE Trans. Vehic. Techno., vol. 56. no. 4, Jul. 2007

[11] Z. Tang, R. C. Cannizzaro, G. Leus and P. Banelli, "Pilot-assisted Timevarying Channel Estimation for OFDM Systems" in IEEE Trans. Signal Process., vol. 55, pp. 2226-2238, May 2007.

[12] L. Rugini, P. Banelli and G. Leus, “ Block DFE and windowing for Doppler-affected OFDM systems" in IEEE Signal Process. Adv. Wireless Commun. (SPAWC), pp. 470-474, Jun. 2005.

[13] B. Yang, K. B. Letaief, R. S. Cheng and Z. Cao, "Channel Estimation for OFDM Transmisson in Mutipath Fading Channels based on Parametric Channel Modeling" in IEEE Trans. Commun., vol. 49, no. 3, pp. 467-479, March 2001.

[14] K. D. Teo and S. Ohno, “ Optimal MMSE Finite Parameter Model for Doubly-selective Channels " in Proc. IEEE GLOBAL COMMUNICATIONS Conf., pp. 3503-3507, Dec.2005.

[15] G. Leus, " On the Estimation of Rapidly Time-Varying Channels " in Euro. Signal Process. Conf. (EUSIPCO), Vienna, Austria, Sept. 2004.

[16] A. R. Kannu and P. Schniter, "MSE-optimal Training for Linear Timevarying Channels" in IEEE ICASSP Conf., pp. 789-792, Mar. 2005.

[17] W. C. Jakes, Microwave Mobile Communications. IEEE Press, 1983.

[18] K. E. Baddour and N. C. Beaulieu, "Autoregressive modeling for fading channel simulation" in IEEE Trans. Wireless Commun., vol. 4, no. 4, pp. 1650-1662, July 2005.

[19] B. Anderson and J. B. Moore, Optimal filtering, Prentice-Hall, 1979. 